EPJ manuscript No.

(will be inserted by the editor)

\title{
Simple quantum graphs proposal for quantum devices
}

\author{
A. Drinko ${ }^{1}$, F. M. Andrade ${ }^{1,2}$, and D. Bazeia ${ }^{3}$ \\ 1 Programa de Pós-Graduação em Ciências/Física, Universidade Estadual de Ponta Grossa, 84030-900 Ponta Grossa, PR, Brazil \\ 2 Departamento de Matemática e Estatística, Universidade Estadual de Ponta Grossa, 84030-900 Ponta Grossa-PR, Brazil \\ 3 Departamento de Física, Universidade Federal da Paraíba, 58051-900 João Pessoa, PB, Brazil
}

Received: date / Revised version: date

\begin{abstract}
The control of the quantum transport is an issue of current interest for the construction of new devices. In this work, we investigate this possibility in the realm of quantum graphs. The study allows the identification of two distinct periodic quantum effects which are related to quantum complexity, one being the identification of transport inefficiency, and the other the presence of peaks of full transmission inside regions of suppression of transport in some elementary arrangements of graphs. Motivated by the power of quantum graphs, we elaborate on the construction of simple devices, based on microwave and optical fibers networks, and also on quantum dots, nanowires and nanorings. The elementary devices can be used to construct composed structures with important quantum properties, which may be used to manipulate the quantum transport.

Published in Eur. Phys. J. Plus 135, 451 (2020). https://doi.org/10.1140/epjp/s13360-020-00459-9
\end{abstract}

PACS. 03.65.-w Quantum mechanics - 03.65.Nk Scattering theory

\section{Introduction}

Since the pioneering work of Linus Pauling [1] in the context of free electrons in organic molecules, quantum graphs have been used to describe the behavior of quantum particles in idealized physical networks [2,3]. In the last twenty years or so, the interest in quantum graphs has increased importantly, mainly because of the richness of the subject, which is related to a variety of issues in physical and mathematical sciences [4-12]. For instance, quantum graphs have been simulated experimentally in microwave networks [4] and it is also possible to synthesize quantum nanowires networks with sequential seeding of branching structures [5] and also, using conventional microfabrication facilities [6]. Many properties and techniques related to the study of quantum graphs and their applications can be found in [7]. Moreover, in the recent literature we noted the interesting investigations [8-10], in which the authors deal with issues on graphs and simulations via microwave networks [8], in a way similar to Ref. [4]. The case of networks of fibers and splitters adds another perspective [9] that consists in the construction of complex active optical network of fibers and splitters, which is further explored in [10] and may be nicely modelled by graphs. Also, quantum graphs formalism has been used to study irregular eletromagnetic cavities [11] and scattering of eletromagnetic waves propagating through networks of cables [12].

Among the several possibilities to describe physical properties of graphs, an interesting procedure concerns the Green's function approach, which was proposed in [13] and further explored in [14, 15]. In this work, we rely on the Green's function approach to investigate scattering properties of quantum graphs. The graphs to be studied below have two leads attached to two distinct vertices that are further connected with distinct edges. The details of the calculations and the use of graphs with the focus on the global transmission coefficients are all given in $[14,16]$, in which we explain and explore carefully the concepts and techniques used to implement the calculations that ended up with the current study. In Ref. [16], in particular, we concentrated mainly on hexagonal graphs with vertices of degree 3 , with ideal leads and edges. We did this to focus mainly on the transmission features of simple ideal graphs described by vertices of degree 3 , in this way circumventing the appearance of effects related with the presence of vertices of distinct degrees. In this work, we deal with the two simplest regular graphs, which contain only two vertices of degree 3 . We investigate these graphs concentrating on the possibility to develop simple devices that engender interesting quantum effects.

The study starts with the calculation of global transmission amplitudes of quantum graphs as a function of the wave number of the incident signal. The focus is on the presence of a periodic effect of transport suppression and on the possibility to identify very narrow peaks of wave numbers in which the transmission coefficient increases significantly inside periodic regions of full suppression of transport. We also examine other effects, in particular, very narrow peaks of full transmission inside regions of full suppression. Here, however, we concentrate on the search of peaks of full transmission that we call peaks of constructive 
(a)

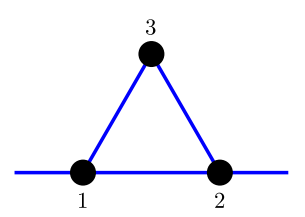

(b)

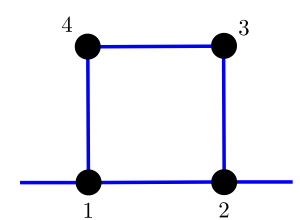

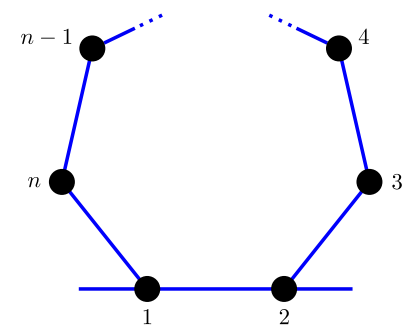

Fig. 1. (Color online) (a) The two quantum graphs to be studied in this work, the regular triangle $\left(C_{3}\right)$ with 3 vertices, and the square $\left(C_{4}\right)$ with 4 vertices. (b) The cycle graph on $n$ vertices.

quantum interference, which appear inside regions of suppression of transmission. Evidently, the identification of those peaks allows for a diversity of applications, including electronic transport in photosynthetic processes that seem to offer a biological advantage [17, 18], and single-molecule quantum transport in break junctions [19], among other possibilities.

We organize the work as follows: in section 2 we use the Green's function approach to obtain the transmission amplitude of some cycle graphs, in particular for the $C_{3}$ and $C_{4}$ graphs shown in Fig. 1(a), and of the composition shown in Fig. 3. In section 3 , we conclude the work with some comments and suggestions of future works.

\section{Results}

The two simple quantum graphs of interest in this work are depicted in Fig. 1(a). They are constructed owing to simplicity having only two vertices of degree 3 , and they conform with the two simplest regular geometric forms, the equilateral triangle and the square, and we refer to them by $C_{3}$ and $C_{4}$, that have three and four equal edges, respectively. We consider the situation where the Schrödinger operator at each edge contains no potential, since we are dealing with ideal edges: $H=-\left(\hbar^{2} / 2 m\right) d^{2} / d x^{2}$, where $\hbar$ is the Planck constant divided by $2 \pi$ and $m$ is the mass of the particle. Then, it is of the free particle type, so the solution has the free particle form. Moreover, we are considering general boundary conditions at the vertices. This means that at the vertex $j$ the quantum amplitudes obey the quantum flux conservation [20]. We can use the above information and the Green's function formalism in [14-16] to write down the general solution for Green's function for the regular $C_{3}$ and $C_{4}$ graphs with edges of the same length $\ell$ and the external leads attached to two neighbor vertices (vertices 1 and 2 in Fig. 1). The Green's function is given by

$$
G_{C_{n}}\left(x_{f}, x_{i} ; k\right)=\frac{m}{i \hbar^{2} k} T_{C_{n}}(k) e^{i k\left(x_{i}+x_{f}\right)}
$$

in which $x_{i}$ and $x_{f}$ are arbitrary points in the entering and exit leads and $n=3,4$.

For the symmetrical case in which the individual transmission amplitudes at the vertices 1 and 2 in $C_{3}$ are equal, namely, $r_{2}=r_{1}=r, t_{2}=t_{1}=t, r_{3}=r^{\prime}$ and $t_{3}=t^{\prime}$ by setting $z=e^{i k \ell}$ the global transmission amplitude for $C_{3}$ is given by

$$
T_{C_{3}}(k)=\frac{t^{2} z}{g_{C_{3}}}\left\{\left[1-(r-t)^{2} z^{2}\right] t^{\prime} z+\left[1-r_{3}(r-t) z^{2}\right]^{2}-(r-t)^{2} t^{\prime 2} z^{4}\right\}
$$

with

$$
g_{C_{3}}=1-r\left(r+2 r^{\prime}\right) z^{2}-2 t^{2} t^{\prime} z^{3}+r\left[r r^{\prime}\left(2 r+r^{\prime}\right)-2 r^{\prime} t^{2}-r t^{\prime 2}\right] z^{4}-(r-t)^{2}(r+t)^{2}\left(r^{\prime}-t^{\prime}\right)\left(r^{\prime}+t^{\prime}\right) z^{6} .
$$

Likewise, for $C_{4}$ in which the individual transmission amplitudes are equal at the vertices 1 and 2 and at the vertices 3 and 4 , namely, $r_{1}=r_{2}=r, t_{1}=t_{2}=t, r_{4}=r_{3}=r^{\prime}$ and $t_{4}=t_{3}=t^{\prime}$, the global transmission is given by

$$
T_{C_{4}}(k)=\frac{t^{2} z}{g_{C_{4}}}\left[1-(r-t)\left(r^{\prime}-t^{\prime}\right) z^{2}\right]\left[1-(r-t)\left(r^{\prime}+t^{\prime}\right) z^{2}\right]\left[1-\left(r^{\prime}-t^{\prime}\right)\left(r^{\prime}+t^{\prime}\right) z^{2}\right],
$$

with

$$
\begin{aligned}
g_{C_{4}}= & 1-\left(r+r^{\prime}\right)^{2} z^{2}+2\left[r r^{\prime}\left(r^{2}+r r^{\prime}+r^{\prime 2}-t^{2}\right)-\left(r r^{\prime}+t^{2}\right) t^{\prime 2}\right] z^{4} \\
& -\left[-r r^{\prime}\left(r+r^{\prime}\right)+r^{\prime} t^{2}+r t^{\prime 2}\right]^{2} z^{6}+\left(r^{2}-t^{2}\right)^{2}\left(r^{\prime 2}-t^{\prime 2}\right)^{2} z^{8} .
\end{aligned}
$$

The above results can be obtained standardly from Refs. [14-16]. If we impose the Neumann-Kirchhoff (NK) boundary condition [7] at all vertices, we have $r=-1 / 3, t=2 / 3$ and $r^{\prime}=0, t^{\prime}=1$ [3]. In this case the global transmission amplitudes simplify and 


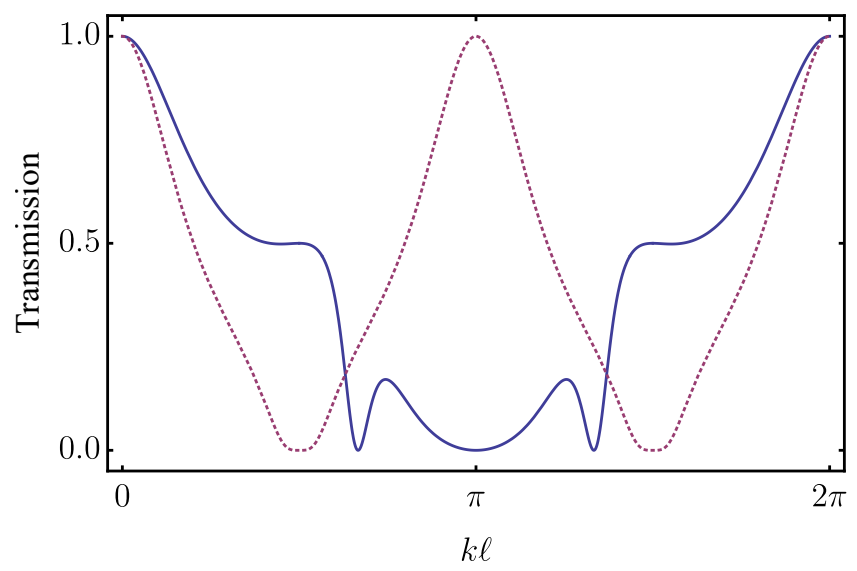

Fig. 2. (Color online) The transmission coefficients of the two possibilities displayed in Fig. 1, with the blue solid curve corresponding to the triangle graph and the violet dotted curve to the square graph using NK boundary conditions.

we can write

$$
\begin{aligned}
T_{C_{3}}(k) & =\frac{4 z\left(1+2 z+2 z^{2}+z^{3}\right)}{9-9 z-8 z^{2}+z^{4}+z^{5}}, \\
T_{C_{4}}(k) & =\frac{4 z\left(1-z^{2}\right)^{2}}{9+8 z-z^{6}} .
\end{aligned}
$$

Actually, for NK boundary condition, it is possible to write an expression for the cycle graph on $n$ vertices, $C_{n}$ (see Fig. 1(b)). The general result is

$$
T_{C_{n}}(k)=\frac{4\left(e^{n i k \ell}-1\right)\left(e^{i k \ell}+e^{(n-1) i k \ell}\right)}{9-e^{2 i k \ell}-e^{2(n-1) i k \ell}-8 e^{n i k \ell}+e^{2 n i k \ell}} .
$$

In Fig. 2 are displayed the transmission coefficient $\left|T_{C_{3}}(k)\right|^{2}$ and $\left|T_{C_{4}}(k)\right|^{2}$. There, one notices that although $\left|T_{C_{4}}(k)\right|^{2}$ (violet dotted curve) has a simpler undulating behavior, the $\left|T_{C_{3}}(k)\right|^{2}$ (blue solid curve) on the contrary shows a richer structure. This is an unexpected result and shows that, despite its geometric simplicity, the triangular arrangement leads to a complex behavior that may induce transport inefficiency. To understand these issues more accurately, we can think of the difference $\left|T_{C_{3}}(k)\right|^{2}-\left|T_{C_{4}}(k)\right|^{2}$ : from Fig. 2 one sees that it can be positive or negative, depending on the wave number of the signal entering the graph, showing that the transmission through the triangle is not always higher than the one of the square.

To add more information on this, let us think of the calculation in terms of the hitting time, which we interpret here as the expected number of steps the signal has to give before leaving the graph at the right or left side, after entering it from the left or right side. The calculation is easier at the classical level, but since we are interested in quantum effects, one implement it at quantum level. The calculation of the hitting time is based on the fact that, in the context of random walks on graphs, the concept of hitting time is directly related to the expected number of steps to reach the vertex $f$ starting from a vertex $i$ [21]. This concept can be extended to the realm of quantum mechanics in the context of quantum walks, which are the quantum version of random walks [22]. So, the corresponding quantity for a quantum walk is the expected number of steps to reach the quantum state in the edge $e_{n}$ starting in the state in the edge $e_{i}[23,24]$. Actually, quantum walks and quantum graphs are deeply related to each other [25] and this relation was further explored in [26]. Based on results of [26], it turns out that the Green's function for a quantum graph is actually a generating function for all the possible walks leaving the entrance lead and getting the exit lead in the scattering process. In this manner, the term $z=e^{i k \ell}$ is equivalent to a time step in the quantum walk problem. So, to extract all the paths with exactly $m$ steps we use the step operator [26]

$$
\hat{S}_{m}=\left.\frac{1}{m !} \frac{\partial^{m}}{\partial z^{m}}\right|_{z=0} .
$$

In this way, the total probability for the quantum walker to leave the entrance lead and to get the exit lead of the graph in exactly $m$ steps in the scattering process is

$$
P(m)=\left|\hat{S}_{m} T_{C_{n}}\right|^{2},
$$

with $T_{C_{n}}$ given by Eqs. (2) and (4). Additionally, the probability to measure the particle for the first time in the exit lead, regardless the number of steps, $P_{\text {out }}$, is given by

$$
P_{\mathrm{out}}=\sum_{m=1}^{\infty} P(m) .
$$




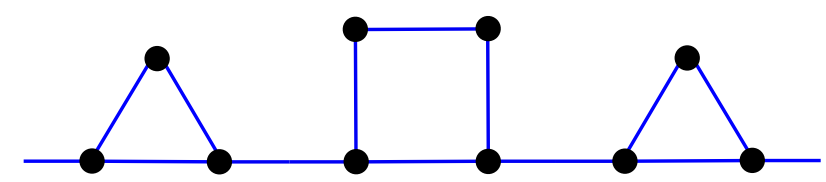

Fig. 3. (Color online) Illustration of the series structure described by the triangle-square-triangle composition.

Then, it is possible to define a conditional hitting time, $h$, as [23, 24]

$$
h=\frac{1}{P_{\text {out }}} \sum_{m=1}^{\infty} m P(m) .
$$

The hitting time corresponding to the two graphs under consideration, using NK boundary conditions, are $h_{C_{3}}=1.91612$ and $h_{C_{4}}=155 / 72=2.15278$, showing that the triangle is expected to be in general more efficient in the quantum transport. However, the effects that are captured by the transmission coefficients of the quantum graphs make the problem more complex, inducing the quantum transport through the triangular graph to follow the unexpected pattern that appear in Fig. 2, giving rise to transport inefficiency in a large region of wave number.

The complexity of the transmission coefficients suggest that we further explore other arrangements. A simple possibility is to arrange the two graphs in series and in parallel, and here we report on the interesting cases in which one adds together the compositions triangle-triangle $\left(C_{3} C_{3}\right)$, square-square $\left(C_{4} C_{4}\right)$, and triangle-square-triangle $\left(C_{3} C_{4} C_{3}\right)$; see Fig. 3 for an illustration of the $C_{3} C_{4} C_{3}$ combination. They are series compositions of two and three elements and, in fact, there are several parallel and series arrangements. Here, however, we focus only on the above mentioned three cases, because they unveil the important effects that we are searching for, in particular, the appearance of very narrow peaks of full transmission inside regions of complete suppression. The transmission coefficients of the $C_{3} C_{3}, C_{4} C_{4}$ and $C_{3} C_{4} C_{3}$ compositions are described by $\left|T_{C_{3} C_{3}}(k)\right|^{2},\left|T_{C_{4} C_{4}}(k)\right|^{2}$, and $\left|T_{C_{3} C_{4} C_{3}}(k)\right|^{2}$, and they are displayed in Fig. 4. We see from these results that the series compositions with two triangles and two squares present bands of full suppression and narrow peaks of full transmission. Similarly, the transmission coefficient of the triple composition triangle-square-triangle has a larger band of full suppression and four narrow peaks of full transmission.

We have studied these peaks of transmission carefully and found that the two at the top panel are located at $\pi \pm 0.91393$, and they have the same width 0.02091 . The four at the middle panel are located at $\pi \pm 1.76182$ and at $\pi \pm 1.37977$ and have the same width 0.00600 . The other four peaks at the bottom panel of Fig. 4 are located at $\pi \pm 1.12611$, with width 0.00804 , and at $\pi \pm 0.43440$, with width 0.00812 . The appearance of peaks of full transmission inside regions of full suppression is another important result, that can be used to manipulate the quantum transport. An interesting possibility is to use compositions of the simple triangular and square devices as filters capable of selecting signals with wave number at some very narrow intervals, controlling the quantum transport at the nanometric scale. The periodicity of the effect is also of interest, since it appears in many distinct regions of wave number having the very same form. Moreover, the results depend on $k \ell$, so one can change $\ell$ to vary the wave number position in the filtering process.

It is of interest to remark that there are other possible studies to be done. In particular, if one thinks of the square, for instance, we can connect the second lead to the second neighbor vertex. Also, we can consider the next polygon, the regular pentagon, and now we can connect the second lead to the first or the second neighbor vertex. Moreover, we can think of the regular hexagon, and here connect the second lead to the first, second or third neighbor vertex, and so on and so forth. We have examined some distinct cases, and found no qualitative difference with the results depicted in Fig. 4. Another remark of interest is that all the transmission coefficients described in this work obey $|T(\pi+k \ell)|^{2}=|T(\pi-k \ell)|^{2}$, for $k \ell \in[0, \pi]$. This appears due to the time reversal symmetry of the quantum graphs that we are examining in this work.

The two quantum graphs depicted in Fig. 1 can be thought of as two elementary devices, so one can probe them following the lines of Ref. [4], in which experimental and theoretical results unveil how microwave networks can simulate quantum graphs. This is an interesting possibility, and is further connected to another very recent investigation [8] on graphs and simulations via microwave networks. Another perspective of current interest is to think of considering networks of fibers and splitters, in a way similar to the recent idea of a lasing network, a LANER [9] which is constructed as a complex active optical network of fibers and splitters, which is further explored in [10]. We think that the above results will foster further interest on microwave networks $[4,8,27]$ and also, on the very recent proposal of networks of fibers and splitters $[9,10]$, in particular, on spatial arrangements of graphs, with the use of more complex topologies.

Another line of research concerns the construction of quantum devices at the nanometric scale, simulating the two quantum graphs with QDs connected by leads. The use of QDs for quantum computing is not new [28], and the more recent works [29, 30] illustrate distinct possibilities to fabricate compositions of two or more QDs in nanowires and in Josephson junctions. Here we suppose that electrons in the incoming lead reach a QD from one side and leave the device through the QD at the outgoing lead on the other side, after interacting with the QDs via the edges that connect them. The flux of matter can be controlled by the chemical potentials of electronic sources that are attached to the left and right leads. The QD composition suggested in [31] will certainly display the presence of the peaks of full transmission that we found in the series composition described in the bottom panel in Fig. 4. Since QDs are mesoscopic devices, the current suggestion works to improve the fabrication of quantum devices at the mesoscopic level, in this sense directly contributing for the manipulation of the quantum transport at the mesoscopic scale. 


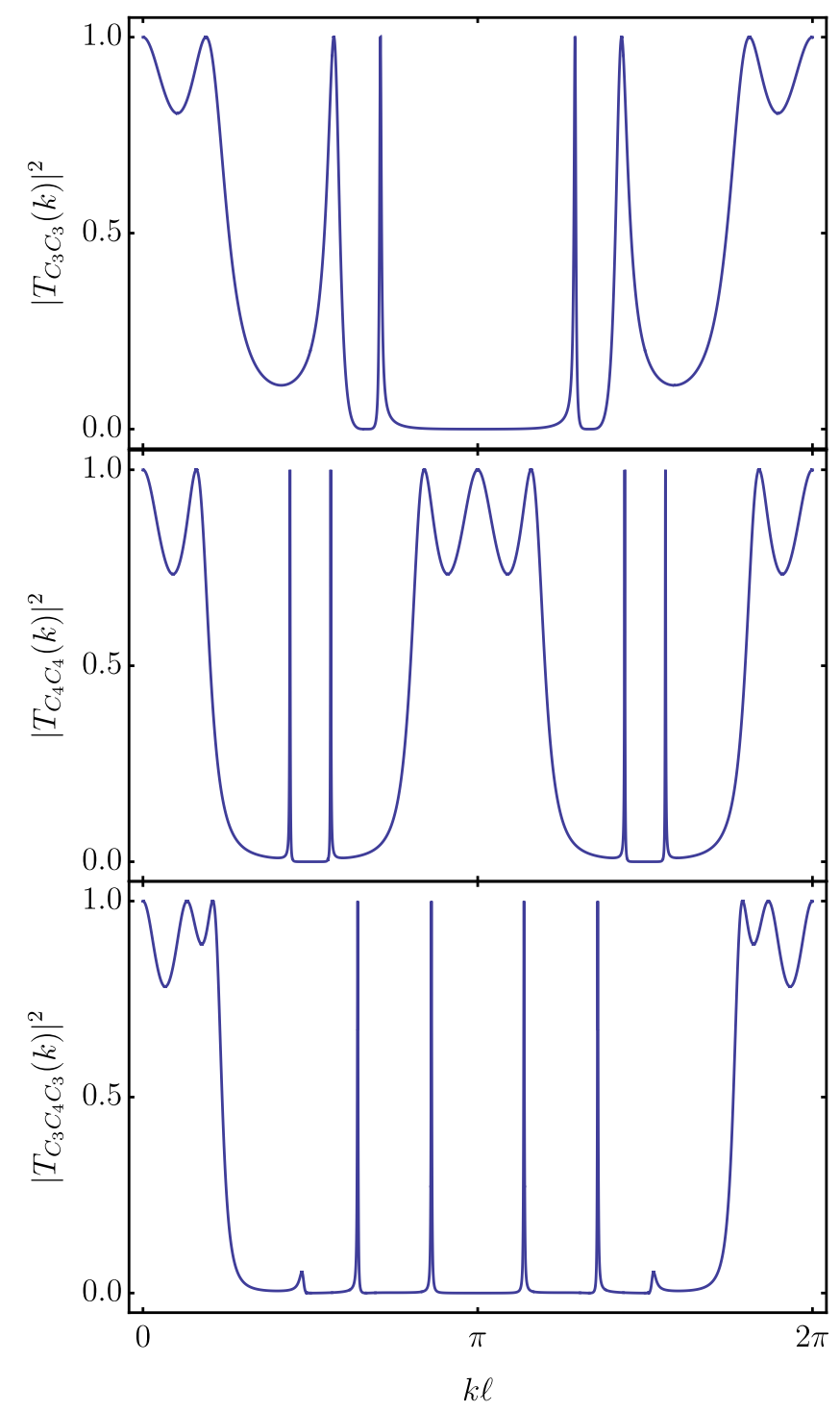

Fig. 4. (Color online) The transmission coefficients of the series arrangements triangle-triangle, square-square and triangle-square-triangle, displayed in the top, middle and bottom panels, respectively.
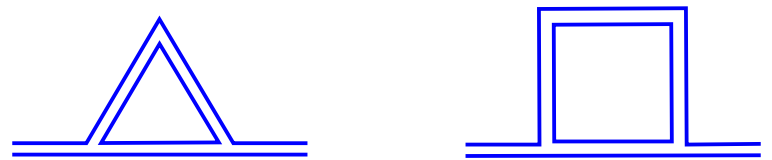

Fig. 5. (Color online) Illustration of the two nanometric devices simulated by the two quantum graphs of Fig. 1.

To implement the above suggestion experimentally, the fabrication of the two elementary devices, the two compositions that requires two QDs connected with two distinct edges is mandatory. They are double QDs and we call them DQDs, and we further require series arrangements of two and three of those DQDs. It can then be noted that the experimental realisation may be intricate, but we additionally observe that instead of QDs, we can use quantum wires or nanowires, which seem to be somehow easier to be implemented experimentally. An interesting line of investigation may follow the experimental implementation developed in [32], concerning the fabrication of single-crystal nanorings of zinc oxide, formed through spontaneous self-coiling process in the growth of polar nanobelts. Other possibilities were accomplished, for instance, in Refs. [33, 34]. Similar ideas were also considered in [35] and in [36], confirming the transport inefficiency in a ringlike disposition of wires of finite width with the addition of an extra central branch in the ring at the nanometric scale. The results provide another strong motivation for the study of the series compositions that we have investigated in this work. Since our graphs are very simple, the quantum devices are also 
very simple. The connection between ringlike quantum devices and quantum graphs is displayed in Fig. 5, with the identification of the two internal arms of the nanometric devices with the internal edges of the quantum graphs shown in Fig. 1. The effects of transport suppression and the presence of peaks of full transmission can be investigated experimentally using very simple microwave networks, similar to the one recently used in [8]. Moreover, since we are dealing with ideal quantum graphs, there is no objection to think of the triangular and square graphs as ringlike structures, with the external leads being attached radially, in the first case forming an angle of $2 \pi / 3$, and in the second an angle of $\pi / 2$. The experimental construction may follow [32-34] and take advantage of the relation required to inscribe equilateral triangles and squares in circles, keeping the (integer) ratios $2 / 1$ and $3 / 1$ between the length of the two internal edges in the triangular and square cases, respectively.

\section{Conclusion}

In this work, we use the Green's function approach to investigate the transmission coefficient of simple quantum graphs and some of their compositions, in particular the composition shown in Fig. 3. It is interesting to note that the transmission coefficient displayed in the bottom panel in Fig. 4 shows a large region of transport suppression, besides the presence of some peaks of full transmission. This kind of behaviour suggests that this structure can be used as a filter for the quantum transport at the nanometric scale.

From the theoretical point of view, the current work motivates several distinct possibilities, an interesting one being the study of graphs with other topologies in the presence of more realistic edges and vertices; see Ref. [14] for more details on how to include different effects on edges and nontrivial boundary conditions on vertices. We can also add appropriate external magnetic fields, which will break the time reversal symmetry and add novel effects in the important case of quantum transport. Another perspective of current interest is to think of the elementary quantum graphs and their series and/or parallel arrangements as molecules. In this case, we can use them to describe quantum transport and interference with the help of mechanical and electrical break junctions, as recently reviewed in Ref. [19, 37]. A particularly important realization may occur with the use of hexagonal arrangements of graphs, for instance, since they can model graphene and other ringlike configurations of current interest. This is now under examination, and we expect to report on the issue in the near future.

\section{Acknowledgements}

This work was partially supported by the Brazilian agencies Conselho Nacional de Desenvolvimento Científico e Tecnológico (CNPq), Fundação Araucária (FAPPR, Grant 09/2016), Instituto Nacional de Ciência e Tecnologia de Informação Quântica (INCT-IQ), and Paraiba State Research Foundation (FAPESQ-PB, Grant 0015/2019). It was also financed by the Coordenação de Aperfeiçoamento de Pessoal de Nível Superior (CAPES, Finance Code 001). FMA and DB also acknowledge CNPq Grants 313274/2017-7 (FMA), 434134/2018-0 (FMA), 306614/2014-6 (DB) and 404913/2018-0 (DB).

\section{References}

1. L. Pauling, J. Chem. Phys. 4, 673 (1936). DOI 10.1063/1.1749766

2. T. Kottos, U. Smilansky, Ann. Phys. (NY) 274, 76 (1999). DOI 10.1006/aphy.1999.5904

3. S. Gnutzmann, U. Smilansky, Adv. Phys. 55, 527 (2006). DOI 10.1080/00018730600908042

4. O. Hul, S. Bauch, P. Pakoński, N. Savytskyy, K. Życzkowski, L. Sirko, Phys. Rev. E 69(5), 056205 (2004). DOI 10.1103/ PhysRevE.69.056205

5. K.A. Dick, K. Deppert, M.W. Larsson, T. Mårtensson, W. Seifert, L.R. Wallenberg, L. Samuelson, Nat. Mater. 3(6), 380 (2004). DOI 10.1038/nmat1133

6. K. Heo, E. Cho, J.E. Yang, M.H. Kim, M. Lee, B.Y. Lee, S.G. Kwon, M.S. Lee, M.H. Jo, H.J. Choi, T. Hyeon, S. Hong, Nano Lett. 8(12), 4523 (2008). DOI 10.1021/nl802570m

7. G. Berkolaiko, P. Kuchment, Introduction to Quantum Graphs (American Mathematical Society, 2012)

8. M. Ławniczak, J. Lipovský, L. Sirko, Phys. Rev. Lett. 122(14), 140503 (2019). DOI 10.1103/physrevlett.122.140503

9. S. Lepri, C. Trono, G. Giacomelli, Phys. Rev. Lett. 118(12), 123901 (2017). DOI 10.1103/physrevlett.118.123901

10. G. Giacomelli, S. Lepri, C. Trono, Phys. Rev. A 99(2), 023841 (2019). DOI 10.1103/physreva.99.023841

11. M. Ahmed, G. Gradoni, S. Creagh, C. Smartt, S. Greedy, G. Tanner, in 2019 International Conference on Electromagnetics in Advanced Applications (ICEAA) (IEEE, 2019), p. 1231. DOI 10.1109/iceaa.2019.8879059

12. M. Ahmed, G. Gradoni, S. Creagh, C. Smartt, S. Greedy, G. Tanner, in 2019 International Symposium on Electromagnetic Compatibility - EMC EUROPE (IEEE, 2019), p. 820. DOI 10.1109/emceurope.2019.8871973

13. A.G.M. Schmidt, B.K. Cheng, M.G.E. da Luz, J. Phys. A 36, L545 (2003). DOI 10.1088/0305-4470/36/42/L01

14. F.M. Andrade, A.G.M. Schmidt, E. Vicentini, B.K. Cheng, M.G.E. da Luz, Phys. Rep. 647, 1 (2016). DOI 10.1016/j.physrep. 2016.07.001 
15. F.M. Andrade, S. Severini, Phys. Rev. A 98(6), 062107 (2018). DOI 10.1103/physreva.98.062107

16. A. Drinko, F.M. Andrade, D. Bazeia, Phys. Rev. A 100(6), 062117 (2019). DOI 10.1103/physreva.100.062117

17. G.S. Engel, T.R. Calhoun, E.L. Read, T.K. Ahn, T. Mančal, Y.C. Cheng, R.E. Blankenship, G.R. Fleming, Nature 446(7137), 782 (2007). DOI 10.1038/nature05678

18. N. Lambert, Y.N. Chen, Y.C. Cheng, C.M. Li, G.Y. Chen, F. Nori, Nat. Phys. 9(1), 10 (2012). DOI 10.1038/nphys2474

19. P. Gehring, J.M. Thijssen, H.S.J. van der Zant, Nat. Rev. Phys. 1(6), 381 (2019). DOI 10.1038/s42254-019-0055-1

20. V. Kostrykin, R. Schrader, J. Phys. A 32(4), 595 (1999). DOI 10.1088/0305-4470/32/4/006

21. J. Kempe, Probab. Theory Relat. Fields 133, 215 (2005). DOI 10.1007/s00440-004-0423-2

22. J. Kempe, Contemp. Phys. 44, 307 (2003). DOI 10.1080/00107151031000110776

23. E. Feldman, M. Hillery, Phys. Lett. A 324, 277 (2004). DOI 10.1016/j.physleta.2004.03.005

24. E. Feldman, M. Hillery, in Coding Theory and Quantum Computing, Contemporary Mathematics, vol. 381, ed. by D. Evans, J. Holt, C. Jones, K. Klintworth, B. Parshall, O. Pfister, H. Ward (2005), Contemporary Mathematics, vol. 381, p. 71

25. G.K. Tanner, in Non-Linear Dynamics and Fundamental Interactions, vol. 213 (Springer-Verlag, 2006), chap. From quantum graphs to quantum random walks, pp. 69-87. DOI 10.1007/1-4020-3949-2_6

26. F.M. Andrade, M.G.E. da Luz, Phys. Rev. A 84(4), 042343 (2011). DOI 10.1103/PhysRevA.84.042343

27. O. Hul, M. Ławniczak, S. Bauch, A. Sawicki, M. Kuś, L. Sirko, Phys. Rev. Lett. 109(4), 040402 (2012). DOI 10.1103/ physrevlett.109.040402

28. D. Loss, D.P. DiVincenzo, Phys. Rev. A 57(1), 120 (1998). DOI 10.1103/physreva.57.120

29. T. Uchida, M. Jo, A. Tsurumaki-Fukuchi, M. Arita, A. Fujiwara, Y. Takahashi, AIP Advances 5(11), 117144 (2015). DOI $10.1063 / 1.4936563$

30. J.E. Saldaña, A. Vekris, G. Steffensen, R. Žitko, P. Krogstrup, J. Paaske, K. Grove-Rasmussen, J. Nygård, Phys. Rev. Lett. 121(25), 257701 (2018). DOI 10.1103/physrevlett.121.257701

31. A.L.R. Barbosa, D. Bazeia, J.G.G.S. Ramos, Phys. Rev. E 90(4), 042915 (2014). DOI 10.1103/physreve.90.042915

32. X.Y. Kong, Y. Ding, R. Yang, Z.L. Wang, Science 303(5662), 1348 (2004). DOI 10.1126/science.1092356

33. F. Martins, B. Hackens, M.G. Pala, T. Ouisse, H. Sellier, X. Wallart, S. Bollaert, A. Cappy, J. Chevrier, V. Bayot, S. Huant, Phys. Rev. Lett. 99(13), 136807 (2007). DOI 10.1103/physrevlett.99.136807

34. B. Hackens, F. Martins, T. Ouisse, H. Sellier, S. Bollaert, X. Wallart, A. Cappy, J. Chevrier, V. Bayot, S. Huant, Nat. Phys. 2(12), 826 (2006). DOI 10.1038/nphys459

35. M.G. Pala, S. Baltazar, P. Liu, H. Sellier, B. Hackens, F. Martins, V. Bayot, X. Wallart, L. Desplanque, S. Huant, Phys. Rev. Lett. 108(7), 076802 (2012). DOI 10.1103/physrevlett.108.076802

36. A.A. Sousa, A. Chaves, G.A. Farias, F.M. Peeters, Phys. Rev. B 88(24), 245417 (2013). DOI 10.1103/physrevb.88.245417

37. N. Xin, J. Guan, C. Zhou, X. Chen, C. Gu, Y. Li, M.A. Ratner, A. Nitzan, J.F. Stoddart, X. Guo, Nat. Rev. Phys. 1(3), 211 (2019). DOI 10.1038/s42254-019-0022-X 